\title{
The Moment Problem in a Certain Function Space of G. G. LORENTZ
}

\author{
By
}

M. S. Ramanujan

In this paper I obtain necessary and sufficient conditions for the existence of a function $f(x)$, belonging to a suitable function space, so that a given sequence of (real) constants $\left\{\mu_{n}\right\}$ may be the sequence of moment constants of the function $f(x)$; i.e. in order that $\mu_{n}$ may have the representation

$$
\mu_{n}=\int_{0}^{1} x^{n} f(x) d x
$$

with $f(x)$ belonging to the specified space of functions. In particular the above problem is solved here for the space $X(C)$ of LoRENTz [2], the definition of which follows in the sequel. For this space, LorENxz has given a solution and we obtain here a different set of conditions in order that (1) may hold, with $f(x) \in X(C)$.

Let $C$ denote a class of positive integrable functions on $[0,1]$ and let $C$ have the following properties:

(i) $1 \in C$;

(ii) $C$ is normal in the sense that if $c_{1}(x) \in C$ and $c_{2}(x)$ is a measurable function such that $0 \leqq c_{2}(x) \leqq c_{1}(x)$ p.p. on $[0,1]$, then $c_{2}(x) \in C$;

(iii) the integrals $\int_{0}^{1} c(x) d x, c \in C$, are bounded.

Evidently, all bounded measurable functions on $[0,1]$ belong to $C$. Now, in relation to $C$ the class $X(C)$ is defined to be the class of all measurable functions $f(x)$ for which

$$
\|f\|=\sup _{c \in C} \int_{0}^{1}|f(x)| c(x) d x<\infty .
$$

It may easily be verified that $X(C)$ is a Banach space (with the above indicated norm) and that $X(C)$ is normal in the sense stated earlier. A suitable choice of $C$ will yield the spaces $L^{p}(p>1), A(\varphi, p)$ and $M(\varphi, p)$, defined by Lorentz [2].

The following definitions are required in the sequel.

Two measurable functions $f(x)$ and $g(x)$, on $[0,1]$, are called rearrangements of each other if, for each real $a$, the sets $[f(x) \geqq a]$ and $[g(x) \geqq a]$ have equal measures. We shall say that the space $X(C)$ has the property of rearrangement invariant norm if $\|\hat{f}\|=\|f\|$ for each rearrangement $\hat{f}(x)$ of $f(x)$. (The spaces $L^{p}, A(\varphi, p)$ etc. cited earlier have this property.) 
We shall, for our investigation, focus our attention on the spaces $X(C)$ which are endowed with the property that the integrals

$$
F(e)=\int_{e} f(x) d x
$$

are such that for each $\varepsilon>0$, there exists a positive $\delta$ in such a manner that (measure of $e) \leqq \delta$ implies $|F(e)| \leqq \varepsilon$, for all $f(x) \in X(C)$ with $\|f\| \leqq 1$. This fact will be briefly denoted by the statement that "the integrals in (2) have the property of uniform absolute continuity".

For the space $X(C)$, which has the property of rearrangement invariant norm and for which the integrals in (2) are uniformly absolutely continuous, LoRENrz [2] has shown that $\mu_{n}$ will have the representation in (1) with $f \in X(C)$ and $\|f\| \leqq M$ if and only if the norms of the functions

$$
f_{n}(x)=(n+1)\left(\begin{array}{l}
n \\
v
\end{array}\right) \Delta^{n-v} \mu_{\nu}, \quad \frac{v}{n+1} \leqq x<\frac{v+1}{n+1}, v=0,1,2, \ldots, n
$$

satisfy the condition $\left\|f_{n}\right\| \leqq M, n=0,1,2, \ldots$

LORENTZ's proof of the above solution rests, among other lemmas, on the uniform approximation of the function $f(x)$, which is continuous in $[0,1]$, by the sequence of Bernstein polynomials $\left\{B_{n}^{f}(x)\right\}$,

$$
B_{n}^{f}(x)=\sum_{v=0}^{n} f\left(\frac{v}{n}\right)\left(\begin{array}{l}
n \\
v
\end{array}\right)(1-x)^{n-v} x^{v}
$$

We shall also employ the same technique as LoREvTz's; however, our starting point is the following lemma, due in substance to MEYER-KönIG and ZELLER [3].

Lemma $\left.1^{1}\right)$. Let $f(x)$ defined in $[0,1]$ be continuous there. Then the sequence $\left\{P_{n}^{f}(x)\right\}$ of Bernstein power series defined by

$$
P_{n}^{f}(x)=\sum_{k=n}^{\infty}\left(\begin{array}{l}
k \\
n
\end{array}\right)(1-x)^{k-n} x^{n+1} f\left(\frac{n+1}{k+1}\right), \quad P_{n}^{f}(0)=f(0)
$$

uniformly approximates $f(x)$ in $[\delta, 1]$, any $\delta>0$.

We need also the following three lemmas.

Lemma 2, Let the space $X(C)$ have the rearrangement invariant norm property. Then each transformation $F(x)=\int_{0}^{1} K(x, t) f(t) d t$, with the properties

$$
\int_{0}^{1}|K(x, t)| d t \leqq A \quad \text { and } \quad \int_{0}^{1}|K(x, t)| d x \leqq A
$$

is a continuous linear operator of norm not exceeding $A$, which maps $X(C)$ into itself.

1) MeYer-König and Zeller [3] do not state lemma 1 in exactly this form. But the content of the lemma follows from a stronger result they prove in Satz 1 of their paper. 
Lemma 3. If the functions $F_{n}(x), 0 \leqq x \leqq 1$, have uniformly absolutely continuous integrals, then there exists a subsequence $F_{n_{k}}(x)$ and an integrable function $F(x)$ such that for each bounded integrable $g$, and $g_{k}(x) \rightarrow g(x)$ uniformly

$$
\int_{0}^{1} F_{n_{k}}(x) g_{k}(x) d x \rightarrow \int_{0}^{1} F(x) g(x) d x .
$$

Also, if the $F_{n}(x)$ all belong to $X(C)$ and $\left\|F_{n}\right\| \leqq 1$, then $F(x) \in X(C)$ and $\|F\| \leqq 1$. Lemmas 2 and 3 are due to LoRENTZ ([2], pp. 79-80).

Lemma 4. Let $\mu_{n}$ be a sequence of real constants. Then

if and only if

$$
\sum_{k=n}^{\infty}\left(\begin{array}{l}
k \\
n
\end{array}\right)\left|\Delta^{k-n} \mu_{n+1}\right| \leqq M \quad(n=0,1,2, \ldots)
$$

$$
\sum_{k=0}^{n}\left(\begin{array}{l}
n \\
k
\end{array}\right)\left|\Delta^{n-k} \mu_{k}\right| \leqq N \quad(n=0,1,2, \ldots) .
$$

Lemma 4 is a consequence of two known theorems, one due to the present author ([4], Theorem 1) and another due to HAUSDORFF ([2], Theorem 3.3.1); a direct proof of lemma 4 is due to Kutriner [1].

Let $\left\{\mu_{n}\right\}$, as before, be a sequence of real constants. We now define a sequence of functions as follows:

$$
f_{n}^{*}(x)=\frac{(k+1)(k+2)}{(n+1)}\left(\begin{array}{l}
k \\
n
\end{array}\right) \Delta^{k-n} \mu_{n+1}, \quad \frac{n+1}{k+2}<x \leqq \frac{n+1}{k+1}, \quad \begin{aligned}
& k=n, n+1, \ldots, \\
& n=0,1,2, \ldots
\end{aligned}
$$

With this definition, we are now in a position to prove our main result.

Theorem. Let the space $X(C)$ have the property of rearrangement invariant norm and let the integrals in (2) be uniformly absolutely continuous. Then in order that the sequence $\mu_{n}(n=1,2, \ldots)$ may have the representation

$$
\mu_{n}=\int_{0}^{1} x^{n} f(x) d x
$$

with $f \in X(C)$ and $\|f\| \leqq M$ it is necessary and sufficient that for each $n,\left\|f_{n}^{*}\right\| \leqq M$.

Proof of the necessity. Let $\mu_{n}$ have the representation $\int_{0}^{1} x^{n} f(x) d x, n=1,2$,
$\ldots$ Then where

$$
f_{n}^{*}(x)=\int_{0}^{1} K_{n}^{*}(x, t) f(t) d t
$$

$$
K_{n}^{*}(x, t)=\frac{(k+1)(k+2)}{(n+1)}\left(\begin{array}{l}
k \\
n
\end{array}\right)(1-t)^{k-n} t^{n+1}, \quad \frac{n+1}{k+2}<x \leqq \frac{n+1}{k+1},
$$

It may easily be verified that

$$
k=n, n+1, \ldots
$$

$$
\int_{0}^{1}\left|K_{n}^{*}(x, t)\right| d t=1 \text { and } \int_{0}^{1}\left|K_{n}^{*}(x, t)\right| d x=1 .
$$


The proof of the necessity is now complete after Lemma 2.

Proof of the sufficiency. Let $f(x)$ be any continuous function in $[0,1]$ and let $P_{n}^{f}(x)$ have the meaning defined in Lemma 1. We shall define $P_{n m}^{f}(x)$ for $n, m=$ $=0,1,2, \ldots$ by

$$
P_{n m}^{f}(x)=\sum_{k=n}^{n+m}\left(\begin{array}{l}
k \\
n
\end{array}\right)(1-x)^{k-n} x^{n+1} f\left(\frac{n+1}{k+1}\right)
$$

with the understanding that $P_{n m}^{f}(0)=f(0)$, for all $n, m$. We make the following preliminary comments.

(i) For each fixed $n$ and $m, P_{n m}^{f}(x)$ is a polynomial in $x$;

(ii) $P_{n m}^{f}(x)$ converges uniformly to $P_{n}^{f}(x)$, as $m \rightarrow \infty$, for $x \in[\delta, 1]$,

(iii) $P_{n}^{f}(x)$ converges uniformly to $f(x)$ in $[\delta, 1]$.

Let us now assume that $\left\|f_{n}^{*}\right\| \leqq M$, for each $n$. Then since $1 \in C$ and

$$
\left\|f_{n}^{*}\right\|=\sup _{c \in C} \int_{0}^{1}\left|f_{n}^{*}(x)\right| c(x) d x
$$

it follows that $\left\|f_{n}^{*}\right\| \leqq M$ implies

$$
\sum_{k=n}^{\infty}\left(\begin{array}{l}
k \\
n
\end{array}\right)\left|\Delta^{k-n} \mu_{n+1}\right| \leqq M
$$

and therefore by Lemma 4, also, that

$$
\sum_{k=0}^{n}\left(\begin{array}{l}
n \\
k
\end{array}\right)\left|\Delta^{n-k} \mu_{k}\right| \leqq N
$$

for each $n$.

Now the polynomials

$$
p(x)=a_{0}+a_{1} x+\cdots+a_{m} x^{m}
$$

with $\left\{a_{n}\right\}$ real and $x \in[0,1]$ form a linear subspace $P[0,1]$ of the space $C[0,1]$ of continuous functions in $[0,1]$. Then when the $\left\{\mu_{n}\right\}$ satisfies the above condition, we have, as shown by LoRENTz ([2], pp. 58-59), that

$$
L(p)=a_{0} \mu_{0}+\cdots+a_{m} \mu_{m}
$$

is a linear form over $P[0,1]$, which can be extended to $C[0,1]$ by setting, for

$$
f \in C[0,1], \quad L(f)=\lim _{n} L\left(f_{n}\right),
$$

where $f_{n}(x)$ is any sequence of polynomials uniformly approximating $f(x)$ in $[0,1]$; also such an extended linear form is continuous over $C$. The same result holds for $[\delta, 1]$ instead of $[0,1]$, for any $\delta>0$.

Thus it follows from the observations made above and from the comments (i) - (iii) that for $x \in[\delta, 1]$, whatever be $\delta>0$, that $L\left(P_{n m}^{j}\right) \rightarrow L\left(P_{n}^{f}\right)$ as $m \rightarrow \infty$ and that $L\left(P_{n}^{f}\right) \rightarrow L(f)$ as $n \rightarrow \infty$. Taking $f(x)=x^{p}, p=0,1,2, \ldots$ we obtain, after a brief 
calculation, that

$$
\sum_{k=n}^{\infty}\left(\frac{n+1}{k+1}\right)^{p}\left(\begin{array}{l}
k \\
n
\end{array}\right) \Delta^{k-n} \mu_{n+1} \rightarrow \mu_{p} \text { as } n \rightarrow \infty .
$$

But the expression on the left hand side above is $\int_{0}^{1} f_{n}^{*}(x) g_{n}(x) d x$ where

$$
g_{n}(x)=\left(\frac{n+1}{k+1}\right)^{p} \quad \text { for } \quad \frac{n+1}{k+2}<x \leqq \frac{n+1}{k+1}, \quad k=n, n+1, \ldots
$$

By an application of Lemma 3 it follows that $\mu_{p}=\int_{0}^{1} x^{p} f(x) d x$ with $f \in X(C),\|f\| \leqq M$.

This completes the proof of the theorem.

Application. In the special case of the space $L^{p}(p>1)$, for example, the condition that $\left\|f_{n}^{*}\right\| \leqq M$ can be expressed in the form

$$
\sum_{k=n}^{\infty}\left[\frac{(k+1)(k+2)}{(n+1)}\right]^{p-1}\left|\left(\begin{array}{l}
k \\
n
\end{array}\right) \Delta^{k-n} \mu_{n+1}\right|^{p} \leqq M, \quad n=0,1, \ldots
$$

while LoRENTz's condition for the same space is

$$
\sum_{k=0}^{n}(n+1)^{p-1}\left|\left(\begin{array}{l}
n \\
k
\end{array}\right) \Delta^{n-k} \mu_{k}\right|^{p} \leqq M, \quad n=0,1, \ldots
$$

Similar conditions for various other special cases of the space $X(C)$ can be derived from the expression that $\left\|f_{n}^{*}\right\| \leqq M$.

It will be interesting to know whether a direct equivalence of the two conditions $\left\|f_{n}\right\| \leqq M$ and $\left\|f_{n}^{*}\right\| \leqq M$, without any appeal to the theory of moment sequences, can be given.

\section{References}

[1] B. Kutrner, Note on a paper by M.S. Ramanujan on quasi-Hausdorff transformations. J. Indian Math. Soc., n. Ser. 21, 97-104 (1957)

[2] G. G. LoRENTz, Bernstein Polynomials. Toronto 1953.

[3] W. Meyer-KönIG und K. ZeLLer, Bernsteinsche Potenzreihen. Studia Math. 19, 89-94 (1960).

[4] M. S. RaManujan, Series-to-series quasi-Hausdorff transformations. J. Indian Math. Soc., n. Ser. 17, 47-53 (1953).

Anschrift des Autors:

M. S. Ramanujan Department of Mathematics

University of Michigan

Ann Arbor (Mich.), USA 